\title{
Functional analysis of cytosolic tryparedoxin peroxidase in antimony-resistant and -susceptible Leishmania braziliensis and Leishmania infantum lines
}

\author{
Juvana M Andrade and Silvane M F Murta
}

\begin{abstract}
Background: Tryparedoxin peroxidase (TXNPx) participates in defence against oxidative stress as an antioxidant by metabolizing hydrogen peroxide into water molecules. Reports suggest that drug-resistant parasites may increase the levels of TXNPx and other enzymes, thereby protecting them against oxidative stress.

Methods: In this study, the gene encoding cytosolic TXNPx (cTXNPx) was characterized in lines of Leishmania (Viannia) braziliensis and Leishmania (Leishmania) infantum that are susceptible and resistant to potassium antimony tartrate $(\mathrm{Sb}(\mathrm{III}))$. We investigated the levels of mRNA and genomic organization of the CTXNPX gene. In addition, we transfected the Leishmania lines with the cTXNPx gene and analysed the susceptibility of transfected parasites to Sb (III) and to hydrogen peroxide $\left(\mathrm{H}_{2} \mathrm{O}_{2}\right)$.
\end{abstract}

Results: Northern blot and real-time reverse transcriptase polymerase chain reaction analyses revealed that the level of TXNPx mRNA was approximately 2.5 -fold higher in the Sb(III)-resistant L. braziliensis line than in the parental line. In contrast, no significant difference in CTXNPx mRNA levels between the L. infantum lines was observed. Southern blot analyses revealed that the CTXNPx gene is not amplified in the genome of the Sb(III)-resistant Leishmania lines analysed. Functional analysis of cTXNPx was performed to determine whether overexpression of the enzyme in L. braziliensis and L. infantum lines would change their susceptibility to Sb(III). Western blotting analysis showed that the level of cTXNPx was 2 to 4-fold higher in transfected clones compared to non-transfected cells. Antimony susceptibility test (EC 50 assay) revealed that L. braziliensis lines overexpressing CTXNPx had a 2-fold increase in resistance to $\mathrm{Sb}$ (III) when compared to the untransfected parental line. In addition, these clones are more tolerant to exogenous $\mathrm{H}_{2} \mathrm{O}_{2}$ than the untransfected parental line. In contrast, no difference in $\mathrm{Sb}$ (III) susceptibility and a moderate index of resistance to $\mathrm{H}_{2} \mathrm{O}_{2}$ was observed in L. infantum clones overexpressing CTXNPX.

Conclusion: Our functional analysis revealed that cTXNPx is involved in the antimony-resistance phenotype in L. braziliensis.

Keywords: L. braziliensis, L. infantum, Drug resistance, Potassium antimony tartrate, Cytosolic tryparedoxin peroxidase, Antioxidant defence

\section{Background}

Leishmaniasis refers to a spectrum of diseases caused by different species of protozoan parasites belonging to the genus Leishmania. An estimated 12 million people are infected with Leishmania parasites and an additional 350 million people are at risk worldwide [1]. The clinical

\footnotetext{
*Correspondence: silvane@cpqrr.fiocruz.br

Centro de Pesquisas René Rachou/FIOCRUZ, Avenida Augusto de Lima 1715, Belo Horizonte 30190-002, MG, Brazil
}

manifestation of the disease depends on genetic factors, the host immune system, and mainly on the parasite species involved [2]. In the New World, L. (Leishmania) infantum (syn. L. (L.) chagasi) [3] is the causative agent of visceral leishmaniasis, whereas L. (V.) braziliensis causes cutaneous and mucocutaneous leishmaniasis [4].

The control of leishmaniasis relies primarily on chemotherapy. The pentavalent antimony-containing compounds (sodium stibogluconate- Pentostam ${ }^{\circ}$ and N-methyl-glucamine- 
Glucantime $^{\odot}$ ) have been the first-line drugs for treatment of all forms of the disease for more than 70 years [5]. The mechanism of action of antimony has not been fully elucidated. Studies suggest that $\mathrm{Sb}(\mathrm{V})$ inhibits macromolecular biosynthesis in amastigotes, possibly altering energy metabolism by inhibiting glycolysis and the oxidative pathway of fatty acids $[5,6]$. $\mathrm{Sb}(\mathrm{III})$ is purported to generate disturbances in the thiol redox potential of the parasite by inducing the efflux of intracellular thiols and by inhibiting trypanothione reductase, resulting in cell death by oxidative stress [7].

The drugs used against leishmaniasis have several drawbacks, including toxic side effects, high cost, and the occurrence of antimony-resistant Leishmania strains [8]. The resistance to pentavalent antimonials has reached epidemic proportions in Bihar (India), where more than $60 \%$ of patients with visceral leishmaniasis were unresponsive to $\mathrm{Sb}(\mathrm{V})$ treatment [9]. Even though the mechanism of antimony-resistance in Leishmania spp. has been widely studied, many questions remain unanswered [8]. It has been described that resistance involves interplay between uptake, efflux, and sequestration of active molecules $[8,10]$.

Most parasites, including Leishmania spp., are more susceptible to reactive oxygen species than their hosts $[8,11]$. To prevent cell damage due to reactive oxygen species (ROS), organisms have developed different antioxidant defence systems [12]. In trypanosomatids, peroxidases display a unique feature in using reducing equivalents derived from trypanothione, a dithiol found exclusively in these protozoa, in contrast to other eukaryotes that utilize glutathione and catalase [13,14]. The function of these antioxidant enzymes include defence against chemical and oxidative stress, by catalyzing the reduction of hydrogen peroxide and small-chain organic hydroperoxides to water and alcohol, respectively. The combined action of trypanothione reductase, tryparedoxin, and tryparedoxin peroxidase is central to the maintenance of a low concentration of hydrogen peroxide $\left(\mathrm{H}_{2} \mathrm{O}_{2}\right)$ [12].

Tryparedoxin peroxidase (TXNPx) belongs to the 2cysteine peroxiredoxin family, and can be grouped according to its compartmentalization to the cytosol or mitochondria [14]. These enzymes are highly conserved and they are present in various Leishmania species [15-17]. Recently, our proteomic analyses have revealed that cytosolic TXNPx (cTXNPx) is overexpressed in antimony-resistant $L$. braziliensis and L. infantum lines [18]. However, its role in the $\mathrm{Sb}(\mathrm{III})$-resistance phenotype in these Leishmania species had not been elucidated. Thus, the aim of the present study was to characterize TXNPx in these lines by assessing mRNA levels and genomic organization. In addition, functional analysis of cTXNPx was performed to determine whether its overexpression in Leishmania lines would change the susceptibility of the parasites to antimony $(\mathrm{Sb}(\mathrm{III}))$ and hydrogen peroxide $\left(\mathrm{H}_{2} \mathrm{O}_{2}\right)$.

\section{Methods}

Leishmania spp. samples

Promastigote forms of L. (Viannia) braziliensis (MHOM/ BR/75/M2904) and L. (Leishmania) infantum (syn. L. (L.) chagasi) (MHOM/BR/74/PP75) were used in this study. $\mathrm{Sb}$ (III)-resistant lines were previously obtained from wildtype $L$. braziliensis and $L$. infantum lines by stepwise increasing the drug pressure with $\mathrm{Sb}(\mathrm{III})$ [19]. These resistant lines are 20 and 4-fold less sensitive to SbIII than their respective parental counterparts [19]. Promastigote forms of these Leishmania lines were grown in M199 medium, harvested in the logarithmic growth phase, washed in PBS and the parasite pellets were used for DNA, RNA and protein preparations.

\section{RNA and DNA preparations}

Total RNA and genomic DNA from Leishmania lines were extracted as previously described [20]. Southern and northern blots were carried out using a protocol previously described [21]. Probes for both assays were prepared by amplification of a 592 bp fragment of cTXNPx gene from L. braziliensis (TritrypDB accession no. Lbr15.1080) using specific primers (forward primer: 5'-CGGTGACGCCAAAATGAAC- 3'; reverse primer: 5'- CTACACCGTGCTGAAGTAGC- 3'). The corresponding fragment has $87.4 \%$ nucleotide sequence identify with the $c T X N P x$ gene from $L$. infantum. The PCR product was labelled with $\left[\alpha-{ }^{32} \mathrm{P}\right]$ dCTP using Nick Translation Kit (Invitrogen, Carlsbald, CA) following the manufacturer's instructions. Blots were hybridized with a ${ }^{32}$ P-labelled $c T X N P x$-specific probe, according to Murta et al. [21]. Band intensities were analyzed using the software CP ATLAS 2.0 (http://lazarsoftware.com/ download.html).

\section{Quantitative real time RT-PCR}

The protocol employed for the preparation of first strand $\mathrm{CDNA}$ and the procedure for real time RT-PCR were as previously described [10]. CDNA was used for RT-qPCR amplification on an ABI Prism 7500 Sequence Detection System (PE Applied Biosystems, Foster City, CA, U.S.A.). The specific primers (forward primer: 5' CGGTGACGCCAAAATGAAC 3'; reverse primer 5' - GAAGTCAAGCGGGTAGAAGAAGAG- 3') employed were designed from the complete nucleotide sequence of the cTXNPx gene (Lbr15.1080). The 18S small subunit ribosomal RNA (18S SSU rRNA) constitutive gene from Leishmania was used to normalize the amount of sample analyzed. The primers (forward primer: 5' - TCTAGGCTACCGTTTCGGCTT-3'; reverse 
primer: 5'-CACACACCGAACCGAAGTTG-3') were designed from the complete nucleotide sequence of the $18 S$ SSU rRNA gene (LmjF.27.rRNA.01). Both pair of primers amplified fragments of $136 \mathrm{bp}$ and $97 \mathrm{bp}$ respectively, in all Leishmania lines (data not shown). Standard curves were prepared for each run using known quantities of pCR 2.1-TOPO plasmids (Invitrogen) containing the $c T X N P x$ and $18 S S S U$ rRNA genes. Estimates of transcript levels were obtained using the Sequence Detection System data analysis software. Values were normalized to those obtained for $18 S$ SSU $r R N A$ for each sample.

\section{Generation of cTXNPX overexpressing lines}

A $600 \mathrm{bp}$ fragment corresponding to L. braziliensis cTXNPx ORF (TritrypDB accession number LbrM15.1080) was amplified with $P f x$ DNA polymerase (Invitrogen) from L. braziliensis genomic DNA using the forward primer: 5 '-tAGATCTccaccATGTCCTGCGGTGACGCCAA-3' and the reverse primer: 5 '-ttAGATCTCTACACCGTGCTGAAGTAGC-3' in which the italicized sequences correspond to BgIII restriction site. The obtained PCR product was cloned into the pGEMT-easy vector (Invitrogen) and subsequently submitted on an ABI 3130 (Applied Biosystems, Foster City, CA, USA) for confirmation of correct sequence. The pGEM-LbcTXNPX construct was restricted with $B g l \mathrm{II}$ and the released fragment was subcloned into the dephosphorylated pIR1-BSD expression vector, generously provided by Dr. Stephen Beverley (Washington University in St. Louis - USA). To confirm correct direction of cloning, the construct was then digested with BamHI releasing fragments that confirmed the sense direction of gene. Thereafter, the constructs pIR1-BSD (empty vector) and pIR1-BSD-Lb cTXNPx were linearized by $S w a \mathrm{I}$ digestion and electroporated into L. braziliensis and L. infantum wild type lines using a GenePulser XCell (BioRad, Hercules, CA, USA). This allowed integration of the vector into the ribosomal small subunit locus [22]. Colonies were obtained following plating on semisolid M199 medium containing Blasticidin (BSD) $(10 \mu \mathrm{g} / \mathrm{ml})$, after 1-2 weeks. Clonal lines were generated and the presence of construct was confirmed by PCR tests using genomic DNA with specific primers for the BSD marker.

\section{Western blotting analysis}

In order to investigate the cTXNPx levels of transfected lines, Western blot assays were carried out. Total protein from the different Leishmania clonal lines were extracted according to the protocol described by Gamarro et al. [23]. Proteins extracts $(20 \mu \mathrm{g})$ were separated by electrophoresis on a $12 \%$ SDS polyacrylamide gel and electrotransferred onto nitrocellulose membrane (Bio-Rad). The membrane was blocked by incubation with $5 \%$ instant non-fat dry milk in PBS supplemented with $0.05 \%$ Tween
20 (PBS-T) for $1 \mathrm{~h}$. The membrane was then washed twice in PBS-T for $5 \mathrm{~min}$ and incubated for $16 \mathrm{~h}$ at $4^{\circ} \mathrm{C}$ in the blocking solution with a polyclonal rabbit anti- $T$. cruzi TXNPx antibody (1:500) [24] (kindly provided by Dr. Fernanda Nogueira, CPqRR, Belo Horizonte, Brazil). The blots were washed three times in PBS-T and then incubated for $1 \mathrm{~h}$ with alkaline phosphatase-conjugated anti-rabbit IgG (Invitrogen) diluted 1:6.000 in blocking solution. Subsequently, the blots were developed using a colorimetric method (Bio-Rad) following the manufacturer's instructions. The blots were normalized using a monoclonal anti- $\alpha$-tubulin antibody (1:10.000) (Sigma, St. Louis, USA). The intensity of the bands was analyzed using the software CP ATLAS 2.0.

\section{Susceptibility of Leishmania spp. clonal lines to $\mathrm{Sb}$ (III) and hydrogen peroxide}

Promastigotes of wild-type L. braziliensis and L. infantum transfected or non-transfected with the constructs pIR1BSD (empty vector) or pIR1-BSD-LbcTXNPx were submitted to $\mathrm{Sb}(\mathrm{III})$ and hydrogen peroxide $\left(\mathrm{H}_{2} \mathrm{O}_{2}\right)$ susceptibility tests. Parasites were incubated in M199 medium at $2 \times 10^{6}$ cells $\mathrm{ml}^{-1}$ in 24-well plates in the absence or presence of various concentrations of SbIII (0.0125 to $1 \mathrm{mg} / \mathrm{ml}$ ) or $\mathrm{H}_{2} \mathrm{O}_{2}$ (200 to $\left.600 \mu \mathrm{M}\right)$ for 48 hours. The concentration of $\mathrm{Sb}$ (III) or $\mathrm{H}_{2} \mathrm{O}_{2}$ required to inhibit the growth by $50 \%\left(\mathrm{EC}_{50}\right)$ was determined using a $\mathrm{Z} 1$ Coulter Counter (Beckman Coulter, Fullerton, CA, USA). EC 50 values were determined from three independent measurements, each performed in triplicate, using the linear interpolation method [25].

\section{Statistic analysis}

All experiments were performed at least three times and data have been represented as mean \pm standard deviation. Data were analyzed by Student's $t$ test performed using the software GraphPad Prism 5.0. A $p$ value of less than 0.05 was considered statistically significant.

\section{Results}

\section{Genomic organization of the cTXNPX gene}

Genomic organization of the $c T X N P x$ gene in $\mathrm{Sb}(\mathrm{III})$ resistant and -susceptible lines of $L$. braziliensis and L. infantum was determined by Southern blot analysis of parasite DNA digested with an endonuclease (EcoRI or BamHI). Hybridization of the blots with an LbcTXNPx gene specific probe revealed that EcoRI-digested DNA gave a major band of $14.0 \mathrm{~kb}$ for both L. braziliensis lines, and bands of $0.6,0.8,4.0$, and $14.0 \mathrm{~kb}$ for L. infantum lines (Additional file 1: Figure S1). Upon hybridization, BamHI-digested DNA from L. braziliensis lines identified a single $12 \mathrm{~kb}$ band, while lines from $L$. infantum contained bands of $0.5,0.6$, and $12 \mathrm{~kb}$ (Additional file 1: Figure S1). Others bands having a low intensity were 
also observed. We observed a polymorphism in the cTXNPx sequence between both Leishmania species analysed. Comparative densitometry of the bands showed no amplification of $c T X N P x$ gene in both $\mathrm{Sb}(\mathrm{III})$-resistant Leishmania lines.

\section{Determination of cTXNPX mRNA levels}

The levels of $c T X N P x$ mRNA across different parasite lines were evaluated by Northern blot. A transcript of $0.5 \mathrm{~kb}$ was detected in Northern blots from $\mathrm{Sb}$ (III)-susceptible and -resistant L. braziliensis and L. infantum lines following hybridization with a ${ }^{32} \mathrm{P}$-labelled cytosolic tryparedoxin peroxidase from L. braziliensis (LbcTXNPx) gene specific probe (Figure 1A). Loading controls using a ribosomal RNA probe are shown in Figure 1B. Densitometry of the transcript profiles revealed that the cTXNPx mRNA level was 2.5-fold higher in the $\mathrm{Sb}$ (III)resistant L. braziliensis line when compared to the parental line (Figure 1C). No difference in the levels of cTXNPx mRNA was detected in both $\mathrm{Sb}(\mathrm{III})$-resistant and -susceptible $L$. infantum lines.

cTXNPx mRNA levels were determined with greater precision by RT-qPCR. The amount of $c T X N P x$ cDNA and $18 S$ SSU rRNA in different Leishmania lines was determined by linear regression analysis using the PCR threshold cycle $\left(\mathrm{C}_{\mathrm{T}}\right)$ values obtained from the standard curve generated with known amounts of the plasmids containing these genes. The amount of $c T X N P x$ cDNA in each line was normalized to the reference housekeeping gene, $18 S$ SSU rRNA (LmjF.27.rRNA.01). The results confirmed the northern blot data, demonstrating that the level of $c T X N P x$ gene transcripts was 2.5 -fold higher in the $\mathrm{Sb}(\mathrm{III})$-resistant L. braziliensis line when compared to the parental line. In addition, no difference was detected between the lines of L. infantum analysed (Figure 1D).

\section{Overexpression of CTXNPX gene in L. braziliensis and L. infantum lines}

We transfected wild-type $L$. braziliensis and L. infantum lines with the construct PIR1-BSD-LbcTXNPx to generate transfectants overexpressing CTXNPx. Linearization of the vector allowed integration of the construct into the ribosomal small subunit locus, by homologous recombination [26]. The successful integration of constructs was confirmed by PCR, using genomic DNA as template and with specific primers for the Blasticidin (BSD) marker. About 24 clones each for pIR1-BSD (empty vector) and pIR1-BSD-LbcTXNPX from L. braziliensis and L. infantum lines were analysed by PCR. It was observed that all blasticidin-resistant clones produced a fragment of $399 \mathrm{bp}$, indicative for the BSD marker (data not shown). These clones were subjected to Western blotting analysis to evaluate the level of cTXNPX. The anti-T. cruzi TXNPX antibody [24] recognized a $23 \mathrm{kDa}$ band in all Leishmania clones (Figure 2A). Densitometry of the cTXNPx band
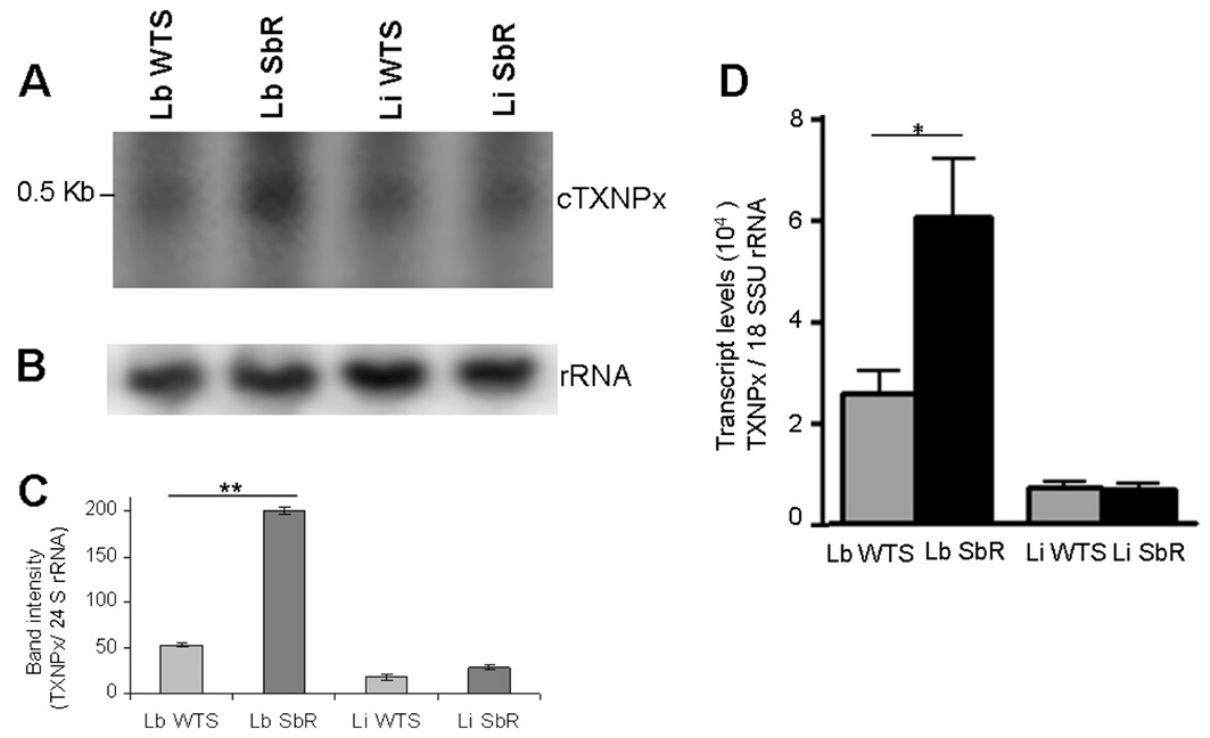

Figure 1 Levels of $c T X N P x$ gene transcript in Sb(III)-susceptible and Sb(III)-resistant $L$. braziliensis and $L$. infantum lines. (A) Northern blot analysis of total RNA $(20 \mu \mathrm{g})$ from Leishmania spp. lines separated on a 1.2\% agarose gel and transferred to nylon membranes. Blots were hybridized with a ${ }^{32}$ P-labelled CTXNPX-specific probe. (B) As a control, the same nylon membrane was hybridized with a ${ }^{32}$ P-labelled 245 rRNA-specific probe. (C) Quantification of bands was done by densitometric analysis using the software CP ATLAS 2.0. (D) Levels of CTXNPX mRNA of $L$. braziliensis and $L$. infantum lines determined quantitatively (relative to the $18 S$ small subunit ribosomal RNA -18S SSU rRNA) by real-time PCR. Mean values of the transcript levels of CTXNPX/SSU \pm standard deviations as determined from three independent experiments are shown. Statistically different values are indicated as follows: ${ }^{*} p<0.04,{ }^{* *} p<0.0002$. 


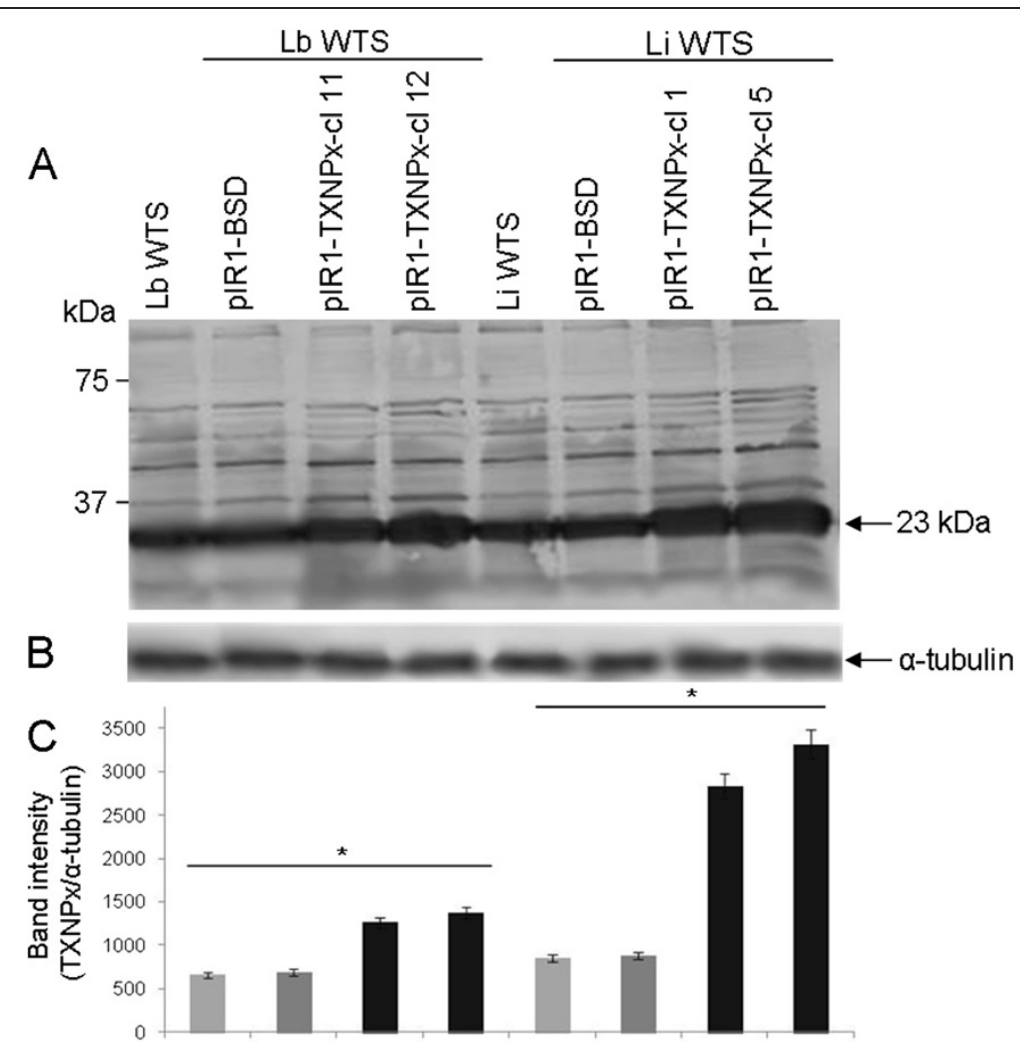

Figure 2 cTXNPx expression levels in clonal lines from $L$. braziliensis and $L$. infantum untransfected or transfected with constructs pIR1-BSD/pIR1-BSD-LbcTXNPx. Total protein $(20 \mu \mathrm{g})$ was separated on a 12\% SDS polyacrylamide gel and blotted onto nitrocellulose membranes. The blots were probed with a polyclonal rabbit anti-T. cruzi CTXNPx antibody (1:500) (A) and with a monoclonal anti-a-tubulin antibody (1:10.000) (B) and developed with NBT/BCIP. (C) Quantification of bands was done by densitometric analysis using the software CP ATLAS 2.0. Statistically different values are indicated as follows: ${ }^{*} p<0.003$.

using an anti- $\alpha$ tubulin antibody as reference (Figure 2B) showed that the level of cTXNPx was 2 to 4-fold higher in the transfected clones from both L. braziliensis and L. infantum lines when compared to the untransfected controls (Figure 2C).

\section{Susceptibility of cTXNPX overexpressing lines to Sb(III)}

In order to investigate whether overexpression of cTXNPx gene favours an antimony-resistance phenotype, clonal lines from $L$. braziliensis and L. infantum transfected with the constructs pIR1-BSD (empty vector) or pIR1-BSDLbcTXNPx and untransfected parasites were subject to a $\mathrm{Sb}$ (III) susceptibility test. As shown in Figure 3A, with increasing concentrations of $\mathrm{Sb}(\mathrm{III})$ there was a rapid decline in the percentage of live parasites in both untransfected and empty vector transfected $L$. braziliensis lines when compared to CTXNPx overexpressing lines. The concentration of $\mathrm{Sb}(\mathrm{III})$ required to inhibit the growth of the parasites by $50 \%$ (effective concentration- $\mathrm{EC}_{50}$ ) was $0.03 \mathrm{mg} / \mathrm{ml}$ for both controls. In contrast, cTXNPx overexpressing lines were 2.3-fold more resistant to $\mathrm{Sb}(\mathrm{III})$, with an $\mathrm{EC}_{50}$ of $0.07 \mathrm{mg} / \mathrm{ml}$. Interestingly, L. infantum cTXNPx overexpressing lines did not show an increase in resistance towards $\mathrm{Sb}(\mathrm{III})$. The cTXNPx overexpressing lines had an EC50 value $(0.11 \mathrm{mg} / \mathrm{ml})$ similar to that of the controls $(0.12 \mathrm{mg} / \mathrm{ml})$ (Figure 3B).

\section{Tolerance of cTXNPx overexpressing lines to hydrogen peroxide}

The tolerance to oxidative stress generated by increased concentrations of hydrogen peroxide was evaluated in the cTXNPx overexpressing lines of L. braziliensis and L. infantum (Figure 4). In vitro assays revealed that the cTXNPx overexpressing LbWTS clones 11 and 12 displayed an $\mathrm{EC}_{50}$ value towards $\mathrm{H}_{2} \mathrm{O}_{2}$ of 408 and $400 \mu \mathrm{M}$, respectively (Figure $4 \mathrm{~A}$ ). In contrast, untransfected and empty vector transfected lines exhibited lower $\mathrm{EC}_{50}$ values for $\mathrm{H}_{2} \mathrm{O}_{2}$ (260 and $256 \mu \mathrm{M}$, respectively). Thus, cTXNPx overexpressing lines were 1.56 -fold $(p<0.001)$ more tolerant to exogenous hydrogen peroxide than controls in L. braziliensis. A moderate index of resistance to $\mathrm{H}_{2} \mathrm{O}_{2}$ was observed for the cTXNPx overexpressing lines of L. infantum compared to that of L. braziliensis. LiWTS clones 1 and 5 displayed $\mathrm{EC}_{50}$ values of 456 and $450 \mu \mathrm{M}$, respectively towards $\mathrm{H}_{2} \mathrm{O}_{2}$ (Figure 4B). In contrast, untransfected and empty vector transfected lines exhibited 

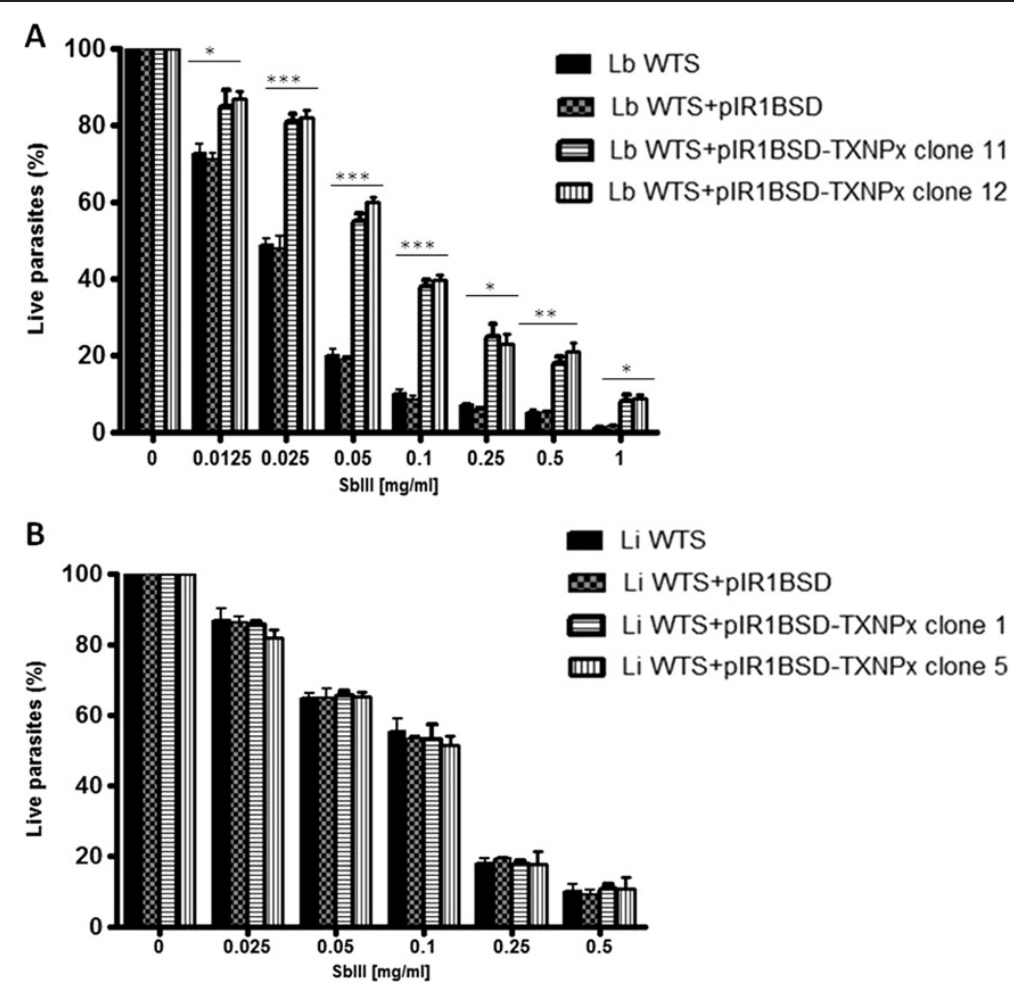

Figure $3 \mathrm{Sb}$ (III) susceptibility of clonal lines from L. braziliensis (A) and L. infantum (B) untransfected or transfected with constructs pIR1-BSD or pIR1-BSD-LbcTXNPx. Parasites were incubated in M199 medium in the absence or presence of various concentrations of Sb(III) $(0.0125$ to $1 \mathrm{mg} / \mathrm{ml})$ for 48 hours and the percentages of live parasites were determined using a Z1 Coulter Counter. Mean values \pm standard deviations from three independent experiments in triplicate are shown. Statistically different values are indicated as follows: ${ }^{*} p<0.04,{ }^{* *} p<0.007$ and ${ }^{* * *} p<0.002$.

lower $\mathrm{EC}_{50}$ values for $\mathrm{H}_{2} \mathrm{O}_{2}$ (373 and $368 \mu \mathrm{M}$, respectively). Thus, cTXNPx overexpressing lines were 1.22 -fold $(p<0.001)$ more tolerant to exogenous hydrogen peroxide than controls in L. infantum.

\section{Discussion}

Antioxidant defence is a promising target for chemotherapy against trypanosomatids, since these organisms present a unique mechanism for detoxification of peroxides that is dependent on trypanothione, which differs from the glutathione-based system found in vertebrates. In trypanosomatids, TXNPx participates in defence against oxidative stress by metabolizing hydrogen peroxide into water molecules [12]. This enzyme is critical to the survival of Leishmania during oxidative stress generated by macrophages and by drugs [27]. In previous studies carried out by our group, using proteomic analysis it was indicated that seven protein spots corresponding to TXNPx were 2 to 5 -fold more abundant in the $\mathrm{Sb}$ (III)-resistant lines of both L. braziliensis and L. infantum species than in their $\mathrm{Sb}(\mathrm{III})$-susceptible parental lines [18]. In the present work, we have extended these results by characterizing cTXNPx in $\mathrm{Sb}(\mathrm{III})$-susceptible and -resistant
L. braziliensis and L. infantum lines and by performing functional analysis of this enzyme.

Our results demonstrated that in the $\mathrm{Sb}(\mathrm{III})$-resistant L. braziliensis line the increase in $c T X N P x$ mRNA levels (2.5-fold) is correlated with high cTXNPx protein levels (3-fold; Matrangolo et al. [18]). In contrast, no difference in the transcription level of this gene was found for $\mathrm{Sb}$ (III)-resistant and -susceptible L. infantum lines. However, Matrangolo et al. [18] showed that the cTXNPx protein level was 1.6-fold higher in the $\mathrm{Sb}(\mathrm{III})$-resistant L. infantum lines when compared with $\mathrm{Sb}(\mathrm{III})$-susceptible parental lines. Since gene expression in trypanosomatids is regulated mainly at the post-transcription level [28], our results suggest that the higher levels of cTXNPx protein detected in the $\mathrm{Sb}$ (III)-resistant L. infantum (LiSbR) line maybe related to an increased stability of mRNA or more efficient protein translation when compared with the wild-type L. infantum (LiWTS) pair.

Altogether, these findings are in agreement with existing data reporting an increase in TXNPx mRNA and TXNPX protein levels in drug-resistant parasites. Nogueira et al. [24] demonstrated an increased expression of TXNPx transcript and TXNPx protein in $T$. cruzi resistant to benznidazole. 

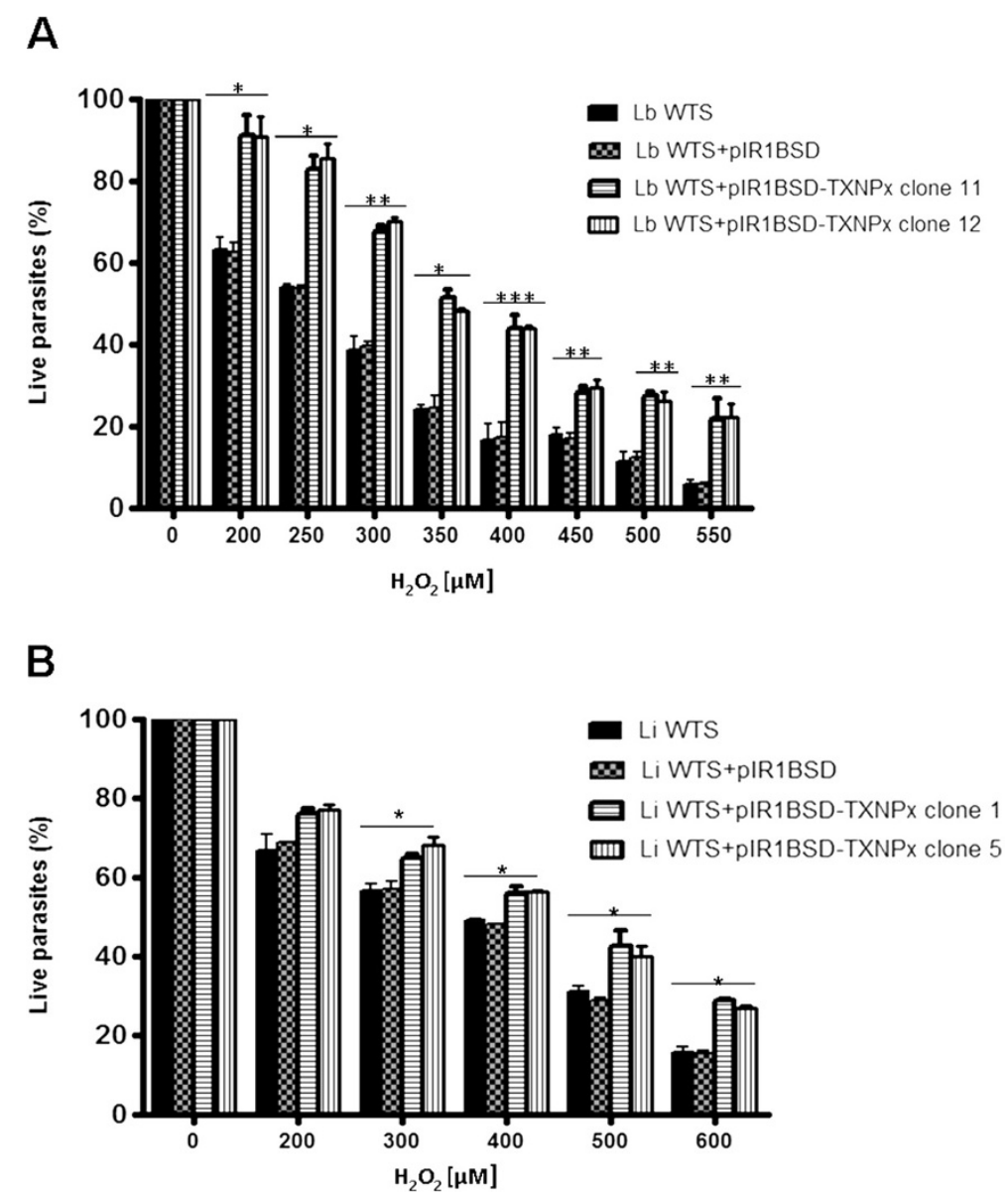

Figure 4 In vitro tolerance of $L$. braziliensis (A) and $L$. infantum (B) lines untransfected or transfected with constructs pIR1-BSD/ pIR1BSD-cTXNPx, to exogenous hydrogen peroxide. Parasites were cultured for $48 \mathrm{~h}$ in the presence of different concentrations of hydrogen peroxide and the percentages of live parasites were determined using a Z1 Coulter Counter. Mean values \pm standard deviations from three independent experiments in triplicate are shown. Statistically different values are indicated as follows: ${ }^{*} p<0.04,{ }^{* *} p<0.005$ and ${ }^{* * *} p<0.0001$.

Metronidazole-resistant Entamoeba histolytica showed a 3-fold increase in its TXNPx mRNA levels [29]. In an Sb (III)-resistant $L$. tarentolae line, an increase of cytosolic (6.5-fold) and mitochondrial (1.8-fold) TXNPx enzymes has been reported [30]. Protein analyses indicated high levels of TXNPx in antimony-resistant $L$. donovani lines [31] and gentamicin-resistant $L$. infantum clones [32].

In order to investigate the role of CTXNPx in protecting the parasite against oxidative stress and its involvement in $\mathrm{Sb}(\mathrm{III})$-resistance, this enzyme was overexpressed in $L$. braziliensis and L. infantum promastigotes. Interestingly, clones from $L$. braziliensis that overexpress the cTXNPx were 2-fold more resistant to $\mathrm{Sb}(\mathrm{III})$. This result indicates that the enzyme is involved in the $\mathrm{Sb}(\mathrm{III})$-resistance phenotype probably along with other enzymes, since the drug resistance phenotype is known to be multifactorial and multigenic. TXNPx is a key antioxidant enzyme important for parasite resistance to oxidative stress. Previous studies have demonstrated that $\mathrm{Sb}(\mathrm{III})$ perturbs the thiol redox potential of the parasite, leading to accumulation of reactive oxygen species (ROS) $[7,33]$. Sb(III) forms a complex with either trypanothione or glutathione that can be sequestrated in an intracellular compartment or directly excreted from parasites in response to SbIII treatment $[7,8]$. Thus, $\mathrm{Sb}$ (III) decreases intracellular thiol buffer capacity, and it also increases the intracellular concentration of the disulfide forms of these thiols through inhibition of trypanothione reductase [7]. These effects of $\mathrm{Sb}$ (III) favour increased levels of ROS. Overexpression of TXPNx confers resistance to $\mathrm{Sb}(\mathrm{III})$ by an increased enzyme activity that acts to reduce levels of ROS induced by exposure to $\mathrm{Sb}(\mathrm{III})$. Data from literature reinforce our results in L. braziliensis, since overexpression of TXNPx in L. tarentolae caused a significant increase in resistance to $\mathrm{Sb}(\mathrm{III})$ [30]. In contrast, overexpression of an enzymatically inactive TXNPx failed to result in resistance to $\mathrm{Sb}(\mathrm{III})$ [30]. These data suggest that the mechanism of TXNPx-dependent resistance is likely due to enhanced antioxidant activity. 
Reports have shown that parasites overexpressing cTXNPx exhibit a high level of resistance to reactive oxygen radicals. In $T$. cruzi, it has been reported that overexpression of TXNPx protects the parasite from $\mathrm{H}_{2} \mathrm{O}_{2}$ and organic peroxide $t$-butyl hydroperoxide damage [34,35]. Lyer et al. [36] observed an increase in the cTXNPx levels in $L$. donovani after exposure to $\mathrm{H}_{2} \mathrm{O}_{2}$. Additionally, the authors also demonstrate that $L$. donovani parasites transfected with cTXNPx are more resistant to antimony and exhibit an increase in virulence when compared to parental parasites. All these data are in agreement with our results showing that cTXNPX overerexpressing L. braziliensis and L. infantum clones are more tolerant to exogenous $\mathrm{H}_{2} \mathrm{O}_{2}$ than an untransfected parental line. However, the $L$. infantum clones present a moderate index of tolerance to $\mathrm{H}_{2} \mathrm{O}_{2}$ when compared to L. braziliensis. This difference could explain, at least in part, the absence of resistance to $\mathrm{Sb}(\mathrm{III})$ in these clones, since our results show that overexpression of this enzyme has no direct involvement in the $\mathrm{Sb}$ (III)-resistance in L. infantum. In addition, the absence of $\mathrm{Sb}(\mathrm{III})$ resistance in cTXNPx overerexpressing L. infantum could also be due to differences in antimonyresistance mechanisms between these two Leishmania species. Moreira et al. [10] demonstrated that an Sb(III)resistant $L$. braziliensis line presented an increased expression of the MRPA gene product and a reduction in the accumulation of antimony. However, no difference was detected between the $\mathrm{Sb}(\mathrm{III})$-resistant and susceptible L. infantum lines.

\section{Conclusion}

The results of the functional analysis revealed that cTXNPx is involved in the antimony-resistance phenotype in L. braziliensis. However, in L. infantum, this enzyme does not seem to be directly associated with resistance to $\mathrm{Sb}(\mathrm{III})$. Interestingly, Wyllie et al. [31] have reported elevated levels of TXNPx in antimonyunresponsive $L$. donovani field isolates. These data suggest that increased expression of this enzyme may play an important role in clinical resistance to antimony.

\section{Additional file}

Additional file 1: Figure S1. Southern blot analysis of the CTXNPX gene from wild-type and Sblll-resistant L. braziliensis and L. infantum lines. Genomic DNA $(10 \mu \mathrm{g})$ was digested with EcoRI (a) and BamHI (b) endonucleases, subject to electrophoresis on a $1 \%$ agarose gel and transferred to nylon membranes. Blots were hybridized with a ${ }^{32}$ P-labeled CTXNPX-specific probe. As control, the same nylon membranes were hybridized with a ${ }^{32} \mathrm{P}$-labeled rRNA-specific probe ( $c$ and $\mathrm{d}$ ). The molecular weight markers used were the $1 \mathrm{~Kb}$ Plus DNA ladder.

\section{Abbreviations}

Sblll: Potassium antimonyl tartrate; WTS: Wild-type susceptible; SbR: SbIll-resistant; Lb: L. (V.) braziliensis; Li: L. (L.) infantum; CTXNPx: Tryparedoxin peroxidase cytosolic;
qRT-PCR: Real time PCR; ROS: Reactive oxygen species; $\mathrm{H}_{2} \mathrm{O}_{2}$ : Hydrogen peroxide; EC: Effective concentration.

\section{Competing interests}

The authors declare that they have no competing interests.

\section{Authors' contributions}

Conceived, designed the experiments, performed the experiments, analyzed the data and helped to draft the manuscript: JMA and SMFM. Both authors read and approved the final manuscript.

\section{Acknowledgements}

The authors wish to thank Dr. Stephen Beverley and Dr. Fernanda Nogueira for kindling provided the pIR1-BSD vector and the polyclonal antibody anti-tryparedoxin peroxidase, respectively and M.Sc. Nayara G. Tessarollo for her assistance in Southern blot assays. We also thank the Program for Technological Development in Tools for Health-PDTIS-FIOCRUZ for use of its facilities. This investigation received financial support from the following agencies: Conselho Nacional de Desenvolvimento Científico e Tecnológico (CNPq/Universal 475782/2012-7), Fundação de Amparo à Pesquisa do Estado de Minas Gerais (FAPEMIG - CBB-PPM 00536/11 and CBB - PPM-00196-13), Coordenação de Aperfeiçoamento de Pessoal de Nível Superior (CAPES), UNICEF/UNDP/World Bank/WHO - TDR and P3D-Programa de descoberta e desenvolvimento de drogas (PROEP/CNPq/FIOCRUZ 401988/2012-0). S.M.F. Murta is a research fellow supported by CNPq (National Council for the Development of Research of Brazil) and J.M. Andrade by CAPES (06563529658/2012).

Received: 9 May 2014 Accepted: 16 August 2014

Published: 29 August 2014

\section{References}

1. World Health Organization: Leishmaniasis. Available at: [http://www.who. int/leishmaniasis/en/] (Last access 03.09.14).

2. Lipoldova M, Demant P: Genetic susceptibility to infectious disease: lessons from mouse models of leishmaniasis. Nat Rev Genet 2006, 7:294-305.

3. Kuhls K, Keilonat L, Ochsenreither S, Schaar M, Schweynoch C, Presber W, Schönian G: Multilocus microsatellite typing (MLMT) reveals genetically isolated populations between and within the main endemic regions of visceral leishmaniasis. Microbes Infect 2007, 9:334-343.

4. Marzochi MC, Marzochi KB: Tegumentary and visceral leishmaniasis in Brazil: emerging anthropozoonosis and possibilities for their control. Cad Saude Publica 1994, 10:359-375.

5. Herwaldt BL: Leishmaniasis. Lancet 1999, 354:1191-1199.

6. Berman JD, Gallalee JV, Best JM: Sodium stibogluconate (Pentostam) inhibition of glucose catabolism via the glycolytic pathway, and fatty acid beta-oxidation in Leishmania mexicana amastigotes. Biochem Pharmacol 1987, 36:197-201.

7. Wyllie S, Cunningham ML, Fairlamb AH: Dual action of antimonial drugs on thiol, redox metabolism in the human pathogen Leishmania donovani. J Biol Chem 2004, 279:39925-39932.

8. Croft SL, Sundar S, Fairlamb AH: Drug resistance in leishmaniasis. Clin Microbiol Rev 2006, 19:111-126.

9. Sundar S: Drug resistance in Indian visceral leishmaniasis. Trop Med Int Health 2001, 6:849-854.

10. Moreira DS, Monte Neto RL, Andrade JM, Santi AMM, Reis PG, Frezard F, Murta SMF: Molecular characterization of the MRPA transporter and antimony uptake in four New World Leishmania spp. susceptible and resistant to antimony. Int J Parasitol Drugs Drug Resist 2013, 3:143-153.

11. Schirmer RH, Schollhammer T, Eisenbrand G, Krauth-Siegel RL: Oxidative stress as a defense mechanism against parasitic infections. Free Radic Res Commun 1987, 3:3-12.

12. Turrens JF: Oxidative stress and antioxidant defenses: a target for the treatment of diseases caused by parasitic protozoa. Mol Aspects Med 2004, 25:211-220.

13. Fairlamb AH, Cerami A: Metabolism and functions of trypanothione in kinetoplastida. Annu Rev Microbiol 1992, 46:695-729.

14. Castro H, Tomas AM: Peroxidases of trypanosomatids. Antioxid Redox Signal 2008, 10:1593-1606. 
15. Levick MP, Tetaud E, Fairlamb AH, Blackwell JM: Identification and characterisation of a functional peroxidoxin from Leishmania major. Mol Biochem Parasitol 1998, 96:125-137.

16. Flohe L, Budde H, Bruns K, Castro H, Clos J, Hofmann B, Kansal-Kalavar S, Krumme D, Menge U, Plank-Schumacher K, Sztajer H, Wissing J, Wylegalla C, Hecht HJ: Tryparedoxin peroxidase of Leishmania donovani: molecular cloning, heterologous expression, specificity and catalytic mechanism. Arch Biochem Biophys 2002, 397:324-335.

17. Castro H, Budde H, Flohe L, Hofmann B, Lunsdorf H, Wissing J, Tomas AM: Specificity and kinetics of a mitochondrial peroxiredoxin of Leishmania infantum. Free Radic Biol Med 2002, 33:1563-1573.

18. Matrangolo FS, Liarte DB, Andrade LC, de Melo MF, Andrade JM, Ferreira RF, Santiago AS, Pirovani CP, Silva-Pereira RA, Murta SM: Comparative proteomic analysis of antimony-resistant and -susceptible Leishmania braziliensis and Leishmania infantum chagasi lines. Mol Biochem Parasitol 2013, 190:63-75.

19. Liarte DB, Murta SMF: Selection and phenotype characterization of potassium antimony tartrate-resistant populations of four New World Leishmania species. Parasitol Res 2010, 107:205-212.

20. Nogueira FB, Krieger MA, Nirde P, Goldenberg S, Romanha AJ, Murta SM: Increased expression of iron-containing superoxide dismutase-A (TcFeSOD-A) enzyme in Trypanosoma cruzi population with in vitro-induced resistance to benznidazole. Acta Trop 2006, 100:119-132.

21. Murta SM, Krieger MA, Montenegro LR, Campos FF, Probst CM, Avila AR, Muto NH, de Oliveira RC, Nunes LR, Nirde P, Bruna- Romero O, Goldenberg S, Romanha AJ: Deletion of copies of the gene encoding old yellow enzyme (TcOYE), a NAD(P)H flavin oxidoreductase, associates with in vitro-induced benznidazole resistance in Trypanosoma cruzi. Mol Biochem Parasitol 2006, 146:151-162.

22. Robinson KA, Beverley SM: Improvements in transfection efficiency and tests of RNA interference (RNAi) approaches in the protozoan parasite Leishmania. Mol Biochem Parasitol 2003, 128:217-228.

23. Gamarro F, Chiquero MJ, Amador MV, Légaré D, Ouellette M, Castanys S: Pglycoprotein overexpression in methotrexate-resistant Leishmania tropica. Biochem Pharmacol 1994, 47:1939-1947.

24. Nogueira FB, Ruiz JC, Robello C, Romanha AJ, Murta SM: Molecular characterization of cytosolic and mitochondrial tryparedoxin peroxidase in Trypanosoma cruzi populations susceptible and resistant to benznidazole. Parasitol Res 2008, 104:835-844.

25. Huber W, Koella JC: A comparison of three methods of estimating EC50 in studies of drug resistance of malaria parasites. Acta Trop 1993, 55:257-261.

26. Goyard S, Beverley SM: Blasticidin resistance: a new independent marker for stable transfection of Leishmania. Mol Biochem Parasitol 2000, 108:249-252.

27. Drummelsmith J, Brochu V, Girard I, Messier N, Ouellette M: Proteome mapping of the protozoan parasite Leishmania and application to the study of drug targets and resistance mechanisms. Mol Cell Proteomics 2003, 2:146-155

28. Vanhame L, Pays E: Control of gene expression in Trypanosomes. Microbiol 1995, 59:223-240.

29. Wassmann C, Hellberg A, Tannich E, Bruchhaus I: Metronidazole resistance in the protozoan parasite Entamoeba histolytica is associated with increased expression of iron containing superoxide dismutase and peroxiredoxin and decreased expression of ferredoxin 1 and flavin reductase. J Biol Chem 1999, 274:26051-26056.

30. Wyllie S, Vickers TJ, Fairlamb AH: Roles of trypanothione S-transferase and tryparedoxin peroxidase in resistance to antimonials. Antimicrob Agents Chemother 2008, 52:1359-1365.

31. Wyllie S, Mandal G, Singh N, Sundar S, Fairlamb AH, Chatterjee M: Elevated levels of tryparedoxin peroxidase in antimony unresponsive Leishmania donovani field isolates. Mol Biochem Parasitol 2010, 173:152-164.

32. Daneshvar H, Wyllie S, Phillips S, Hagan P, Burchmore R: Comparative proteomics profiling of a gentamicin-attenuated Leishmania infantum cell line identifies key changes in parasite thiol-redox metabolism. J Proteomics 2012, 75:1463-1471.

33. Mandal G, Wyllie S, Singh N, Sundar S, Fairlamb AH, Chatterjee M: Increased levels of thiols protect antimony unresponsive Leishmania donovani field isolates against reactive oxygen species generated by trivalent antimony. Parasitology 2007, 134:1679-1687.

34. Finzi JK, Chiavegatto CW, Corat KF, Lopez JA, Cabrera OG, Mielniczki-Pereira AA, Colli W, Alves MJ, Gadelha FR: Trypanosoma cruzi response to the oxidative stress generated by hydrogen peroxide. Mol Biochem Parasitol 2004, 133:37-43.
35. Wilkinson SR, Temperton NJ, Mondragon A, Kelly JM: Distinct mitochondrial and cytosolic enzymes mediate trypanothione-dependent peroxide metabolism in Trypanosoma cruzi. J Biol Chem 2000, 275:8220-8225.

36. Lyer JP, Kaprakkaden A, Choudhary ML, Shaha C: Crucial role of cytosolic tryparedoxin peroxidase in Leishmania donovani survival, drug response and virulence. Mol Microbiol 2008, 68:372-391.

doi:10.1186/1756-3305-7-406

Cite this article as: Andrade and Murta: Functional analysis of cytosolic tryparedoxin peroxidase in antimony-resistant and -susceptible Leishmania braziliensis and Leishmania infantum lines. Parasites \& Vectors 2014 7:406.

\section{Submit your next manuscript to BioMed Central and take full advantage of:}

- Convenient online submission

- Thorough peer review

- No space constraints or color figure charges

- Immediate publication on acceptance

- Inclusion in PubMed, CAS, Scopus and Google Scholar

- Research which is freely available for redistribution

Submit your manuscript at www.biomedcentral.com/submit
C) Biomed Central 